\title{
Intra-class Recognition of Fruits using Color and Texture Features with Neural Classifiers
}

\author{
Susovan Jana \\ School of Education Technology \\ Jadavpur University \\ Kolkata, India
}

\author{
Ranjan Parekh \\ School of Education Technology \\ Jadavpur University \\ Kolkata, India
}

\begin{abstract}
Intra-class recognition of fruits using image processing and pattern recognition techniques, is a challenging task mainly because sub-types of the same fruit show a large amount of similarities between each other and hence more difficult to distinguish than when different types of fruits are involved (inter-class). The problem becomes more acute when the camera viewpoint also changes which tend to change the known characteristics of the fruits like contour shape. To solve this problem, this paper proposes a view point invariant solution for intra-class recognition of fruits by combining color and texture features and using a Neural Network (NN) classifier. Experimentations done on a dataset of 270 fruit images show satisfactory performance across different fruit types and sub-types.
\end{abstract}

\section{General Terms}

Pattern Recognition, Image Processing

\section{Keywords}

Color Histogram, Texture features, Gray Level Co-occurrence Matrix, Neural Network

\section{INTRODUCTION}

Classification of fruits is repetitive and time consuming when done manually [1]. It also requires skilled personnel and in many counties there are shortages of skilled manpower for various reasons. In 2013, India was ranked second in terms of fruit production in the world, just after China [2]. This has created huge demands for automating the classification and sorting of fruits effectively and efficiently, in super market and agro packaging industries in India. In recent years, image processing and computer vision techniques have been applied successfully for automating fruit and vegetable analysis [3]. Developed systems are able to classify fruit classes like Orange, Strawberry and Apple with good performance [4]. But classification of various classes of a same fruit (intraclass) is harder than inter fruit classification because classification features are very similar to each other and difficult to distinguish. In this work, a view point invariant and fruit independent method has been proposed to recognize sub-classes of the same fruit using a combination of texture and color features. The method has been tested on 3 different fruit types and 3 sub-classes of each fruit. To show the effectiveness of the proposed method, sub-classes of fruits very similar to each other with respect to color, shape and texture have been chosen. The paper has been structured as follows. Section 2 describes the related previous works. Section 3 contains detailed descriptions of the proposed method. Experimentation and results are reported in Section 4 followed by the analysis of results in section 5. Finally, section 6 concludes the work with future scopes.

\section{PREVIOUS WORKS}

There are a number of works related to fruit classification. A classification system has been developed to classify 3 classes of Indian mango with object contour model, region based descriptor, and boundary based descriptor \& Bayes classifier [1]. It has been tested on mango classes like Alfonza, Totapuri, and Rumani. Considering the market of Date fruit in Middle East, auto identification of Date fruit for sorting has been proposed to reduce manual effort [5]. The authors have experimented with 7 classes of Date fruits, with color, shape, size and texture related features. Experimentations with Nearest Neighbor, Discriminant Analysis and Neural Networks have been reported. An automatic fruit classification system has been developed for discriminating between varieties of apple, orange and strawberry, varying in shape and color, using Scale Invariant Feature Transform (SIFT) and classifiers like K-NN, SVM and Random Forest [4]. In [6] an SVM based fruit and vegetable classification has been proposed. A single histogram feature has been considered with the combination of sum and difference histograms for each color channel. A vegetable classification system for blind persons to identify vegetable in super market grocery has been proposed in [7] using a combination of color and texture features. Recognition of occluded fruits in natural environment is addressed by some researchers [8]. Image of apples have been used for experimentations with Otsu's segmentation technique [9] to differentiate them from natural environments where the fruits have been occluded and overlapped by branches and leaves. Recognition of clusters of fruits is a challenging job in the field of image processing. A clustered tomato recognition technique has been proposed and tested based on mathematical morphology [10]. In their algorithm firstly, the authors identify tomato with its color then the clustered region is identified by length of the longest edge of the minimum enclosing rectangle. After that erosion has been applied to separate each tomato using an iterative process.

\section{PROPOSED METHOD}

In the previous works, intra-class recognition of fruits has been performed mainly using contour shape features. However the shape can change depending on the view point of the camera with respect to the object of interest. Moreover, some fruits like grapes, are always available in bunches, and extracting individual shapes from a bunch captured at various viewing angles, is a non-trivial task. To address these problems the present work uses a combination of color and texture for intra-class recognition. Fig. 1 depicts the process flow diagram of the proposed method and has been detailed below. 


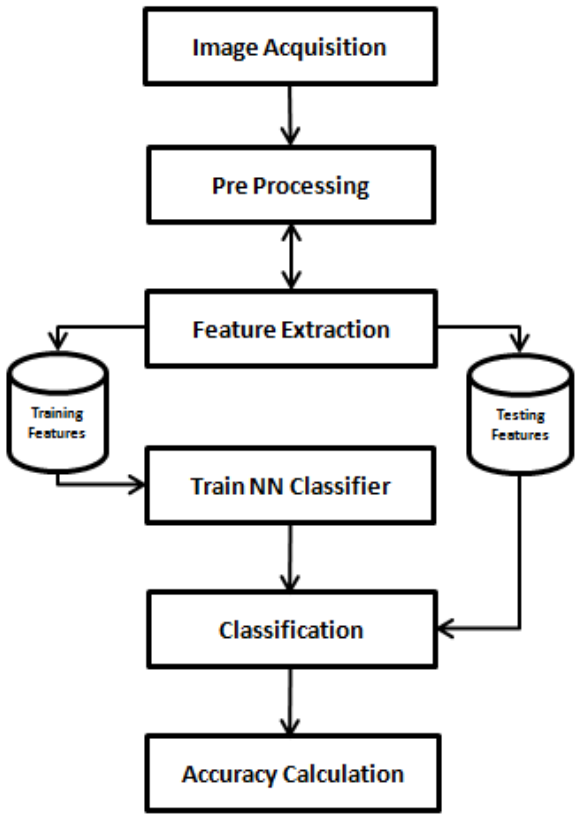

Fig. 1: Process Flow Diagram of Proposed Method

\subsection{Acquisition and Pre-Processing}

The images are captured using a digital camera under controlled lighting conditions and maintaining a fixed distance between the camera and object of interest. Subsequently to reduce computational loads, the images are all resized to standard dimensions.

\subsection{Feature Extraction}

After the completion of pre possessing, both color and texture features have been extracted and used to train a neural classifier for recognition.

Color Features: Color image is split into 3 separate color channels R, G, B and histograms are computed from each channel. The histogram of a digital image with $N$ gray levels is a discrete function $h\left(r_{k}\right)=n_{k}$, where $r_{k}$ is the $k$-th gray level and $n_{k}$ is the number of pixels in the image having gray level $r_{k}$. To denote the three color channels, the symbols $n_{r k}, n_{g k}, n_{b k}$ are being used respectively. Standard deviations of histograms $\sigma_{r}, \sigma_{g}, \sigma_{b}$ are computed separately for each color channel as denoted in Eq. (1) to (3). Here $\mu_{r}, \mu_{g}, \mu_{b}$ denote the mean values.

$\sigma_{r}=\sqrt{\frac{\sum_{k=0}^{N-1}\left(n_{r k}-\mu_{r}\right)^{2}}{N}}$

$\sigma_{g}=\sqrt{\frac{\sum_{k=0}^{N-1}\left(n_{g k}-\mu_{g}\right)^{2}}{N}}$

$\sigma_{b}=\sqrt{\frac{\sum_{k=0}^{N-1}\left(n_{b k}-\mu_{b}\right)^{2}}{N}}$

For each image the color features are represented by a 3element vector comprising of the three standard deviation terms:

$$
F C=\left\{\sigma_{r}, \sigma_{g}, \sigma_{b}\right\}
$$

Texture Features: Statistical texture features are computed from a set of gray-level co-occurrence matrices (GLCM). A GLCM indicates probability of a gray level $i$ occurring in the neighbourhood of another gray level $j$ at a given distance $d$ and angle $\theta$, provided the total number of gray levels $N$ is known. Formally,

$G=\operatorname{Pr}(i, j \mid d, \theta, N)$

GLCMs can be computed along four directions viz. along horizontal $\left(0^{\circ}\right)$, left-diagonal $\left(45^{\circ}\right)$, vertical $\left(90^{\circ}\right)$ and rightdiagonal $\left(135^{\circ}\right)$. A GLCM is made symmetrical by adding it to its transpose and normalized by dividing each element by the sum of all elements. Since dimension of a GLCM can be potentially very large depending on the number of gray levels (e.g. $256 \times 256$ ), they are not directly used in feature computations. Rather a number of scalar features are computed from the GLCMs to provide indications of texture properties inherent within them. Four such scalar features are used here namely, Contrast $(\mathrm{Ct})$, Correlation $(\mathrm{Cn})$, Energy $(E y)$ and Homogeneity $(H y)$ as defined below. Here $S(i, j)$ represents the $(i, j)$-th element of a symmetrical normalized directional GLCM, and $i, j$ each ranges from 1 to $N$.

$$
\begin{aligned}
& C t=\sum_{i, j}|i-j|^{2} S(i, j) \\
& C n=\sum_{i, j} \frac{(i-\mu i)(j-\mu j) S(i, j)}{\sigma_{i} \sigma_{j}} \\
& E y=\sum_{i, j} S(i, j)^{2} \\
& H y=\sum_{i, j} \frac{S(i, j)}{1+|i-j|}
\end{aligned}
$$

For each image the texture feature consists of the four statistical features calculated along four directions $\left(0^{\circ}, 45^{\circ}\right.$, $90^{\circ}, 135^{\circ}$ ) leading to a total of 16 features viz.

$$
\begin{gathered}
F T=\left\{C t_{0^{\circ}}, C t_{45^{\circ}}, C t_{90^{\circ}}, C t_{135^{\circ}},\right. \\
C n_{0^{\circ}}, C n_{45^{\circ}}, C n_{90^{\circ}}, C n_{135^{\circ}}, E y_{0^{\circ}}, E y_{45^{\circ}}, \\
\left.E y_{90^{\circ}}, E y_{135^{\circ}}, H y_{0^{\circ}}, H y_{45^{\circ}}, H y_{90^{\circ}}, H y_{135^{\circ}}\right\}
\end{gathered}
$$

Combined Features: In general fruit classes are identified and recognized by their color, texture and shape. To make the recognition process viewpoint invariant, only on the color and texture traits have been focused on. To represent both of these simultaneously a combination of both of these features has been used. This is especially important for distinguishing subclasses of fruits which might be similar to each other with respect to their color or texture but not both. To make a combined feature the 3-element color vector is concatenated with the 16-element texture vector to generate a 19-element combined vector $F$.

$$
F=\{F C, F T\}
$$

\subsection{Classifier}

A fruit sub-class $i$ consists of a set of $n$ member images. Each sub-class is characterized by the collection of its feature values $T_{i}$ obtained during a training phase. A test image $S$ is said to belong to a specific sub-class if the probability of its feature values belonging to a training class is maximum. Since there are no mathematical models on the basis of which data samples may be classified, classification needs to be done solely on the basis of a set of observations. To establish a 
mapping between the input features and the class memberships, a multi-layered perceptron using a back propagation algorithm (MLP) has been used. Half of the images of a dataset are fed to the neural network to allow it to learn the characteristic color and texture of each class, while the other half is subsequently treated as unknown test samples to evaluate the performance of the system. The recognition accuracy results are reported in the experimentations section.

\section{EXPERIMENTATIONS \& RESULTS}

To test the performance of the proposed system, experimentations were performed on a dataset of 270 images of three fruit types: Mango, Apple and Grapes. Each of the

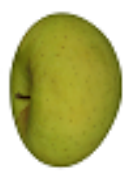

(a)

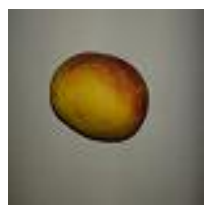

(g)

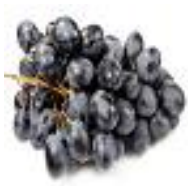

(m)

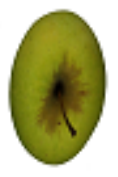

(b)

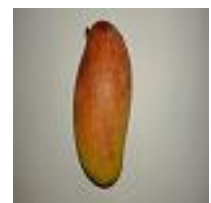

(h)

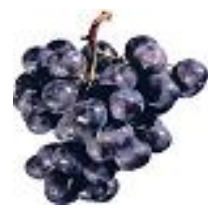

(n)

fruit types is divided into three sub-classes with 30 images in each. Thus there are three types of apples (golden, red delicious, royal), three types of mangoes (gulab khas, himsagar, langra), and three types of grapes (black, red, white). Samples of the training and testing dataset are shown in Fig. 2 and Fig. 3 respectively, with two images for each sub-class. The sub-classes are denoted as A, B, C for each fruit. Most of the digital images are captured with digital camera of a smart phone and the rest are downloaded from websites [11]. To introduce sufficient variations, the images are depicted from different viewpoints and angles. As part of the pre-processing step each image is resized to standard dimensions of $200 \times 200$ and stored in JPG format.

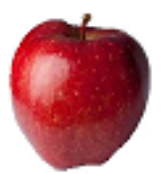

(c)

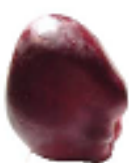

(d)

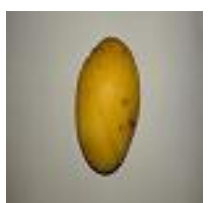

(i)

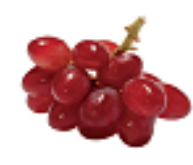

(o)

(j)

(p)

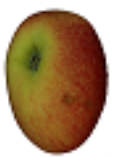

(e)
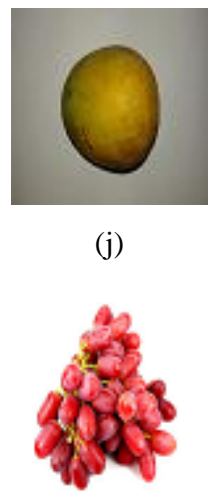

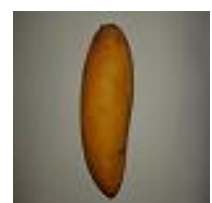

(k)

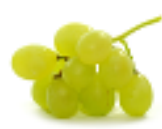

(q)

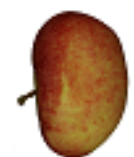

(f)

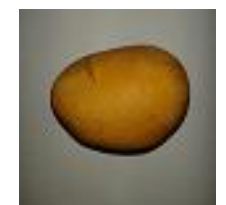

(1)

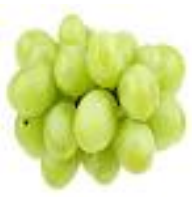

(r)

Figure 2: Training Samples : Apple sub-classes A(a, b), B(c, d), C(e, f); Mango sub-classes : A(g, h), B(i, j), C(k, l); Grape subclasses : $\mathbf{A}(\mathbf{m}, \mathbf{n}), \mathbf{B}(\mathbf{o}, \mathbf{p}), \mathbf{C}(\mathbf{q}, \mathbf{r})$

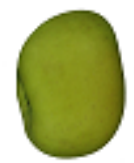

(a)

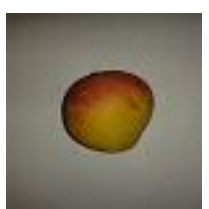

(g)

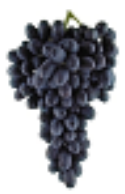

(m)

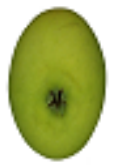

(b)

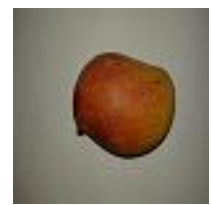

(h)

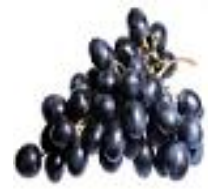

(n)

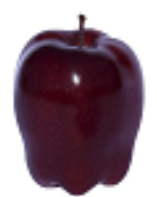

(c)

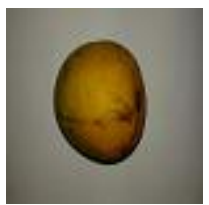

(i)

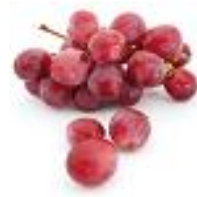

(o)

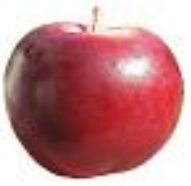

(d)

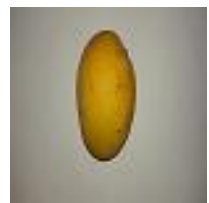

(j)

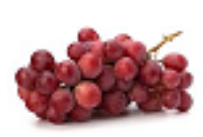

(p)

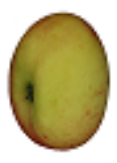

(e)

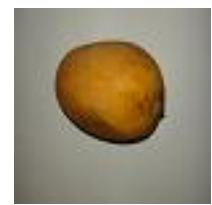

(k)

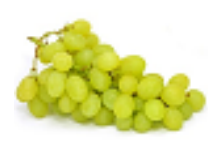

(q)

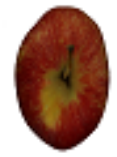

(f)

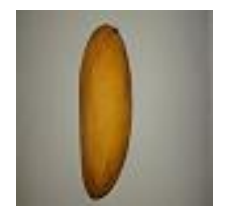

(1)

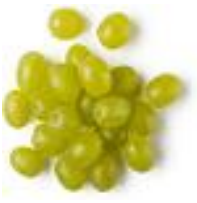

(r)

Figure 3: Testing Samples; Apple sub-classes A(a, b), B(c, d), C(e, f); Mango sub-classes : A(g, h), B(i, j), C(k, l); Grape subclasses : A(m, n), B(o, p), C(q, r) 
Fig. 4 depicts the variations of the three color features $\sigma_{r}, \sigma_{g}, \sigma_{b}$ for the training set members of the three sub-classes A, B, C of the Apple dataset, Fig. 5 depicts the same variations for the Mango dataset, and Fig. 6 for the Grape dataset. Fig. 7 depicts the variation of mean values of contrast (GC), correlation (GN), energy (GE), homogeneity (GH) averaged from the four directional GLCMs computed from the 3 sub-classes $(A, B, C)$ of the Apple training set. Fig. 8 depicts the variation of mean values of texture features for the Mango training set and Fig. 9 those of the Grape training set.
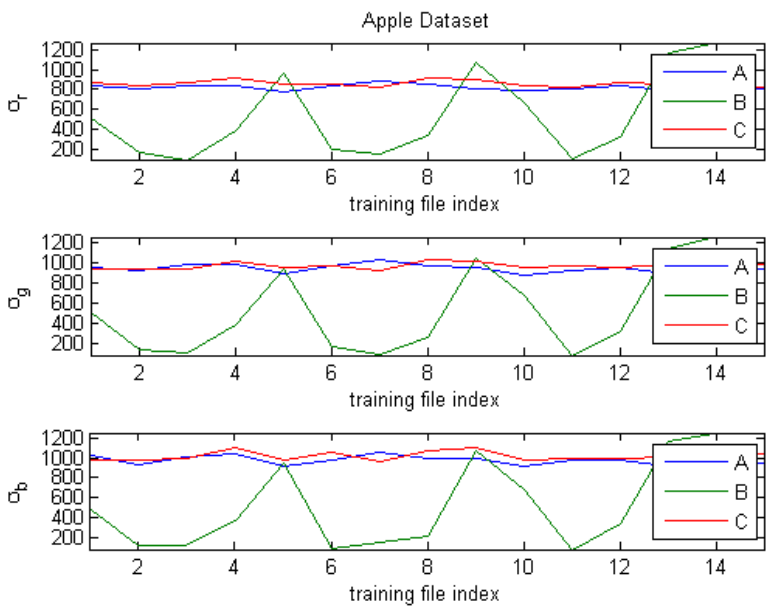

Figure 4: Variation of color features for Apple training set
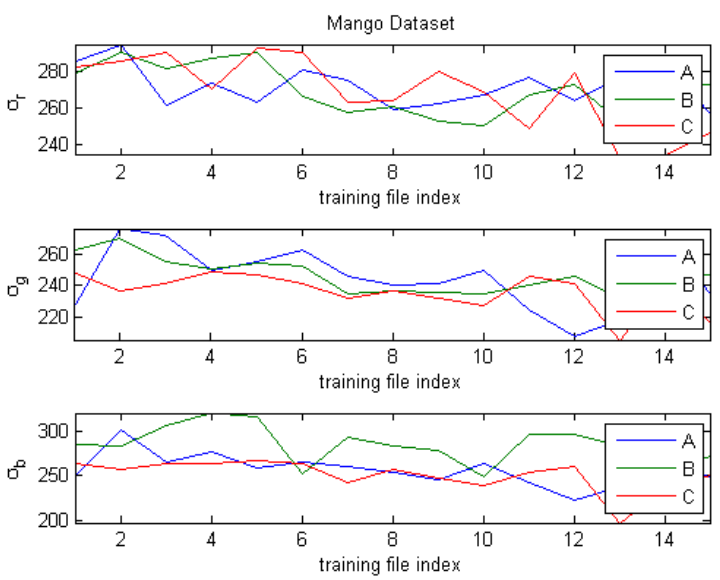

Figure 5: Variation of color features for Mango training set
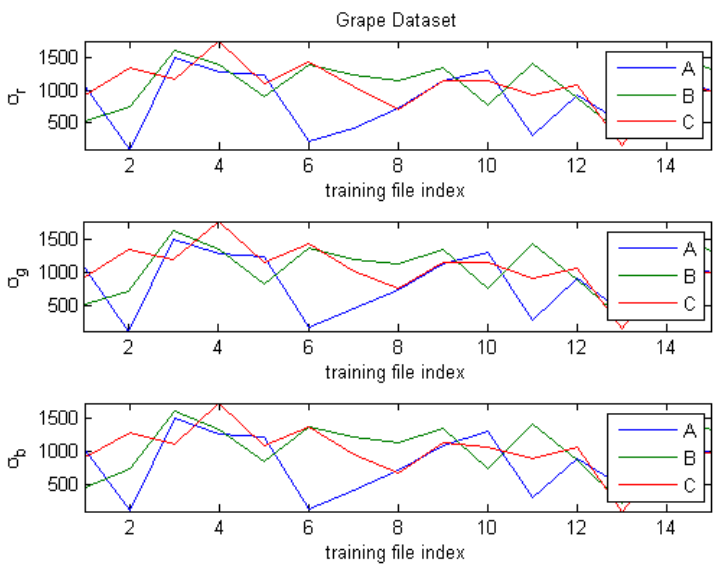

Figure 6: Variation of color features for Grape training set
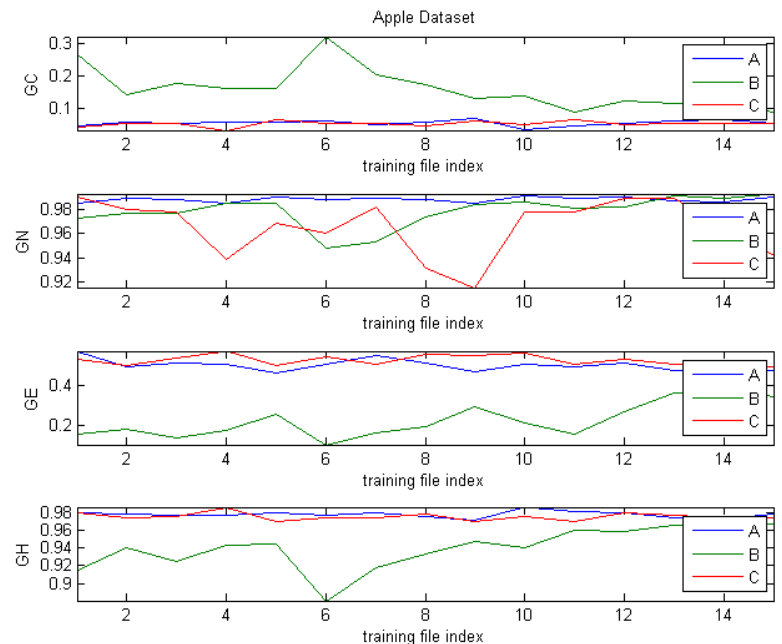

Figure 7: Variation of texture features for Apple training set
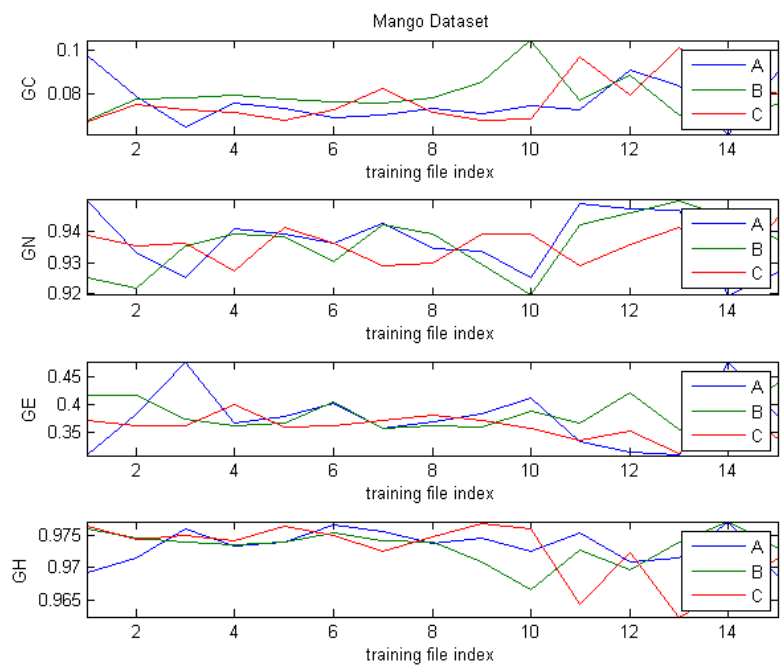

Figure 8: Variation of texture features for Mango training set
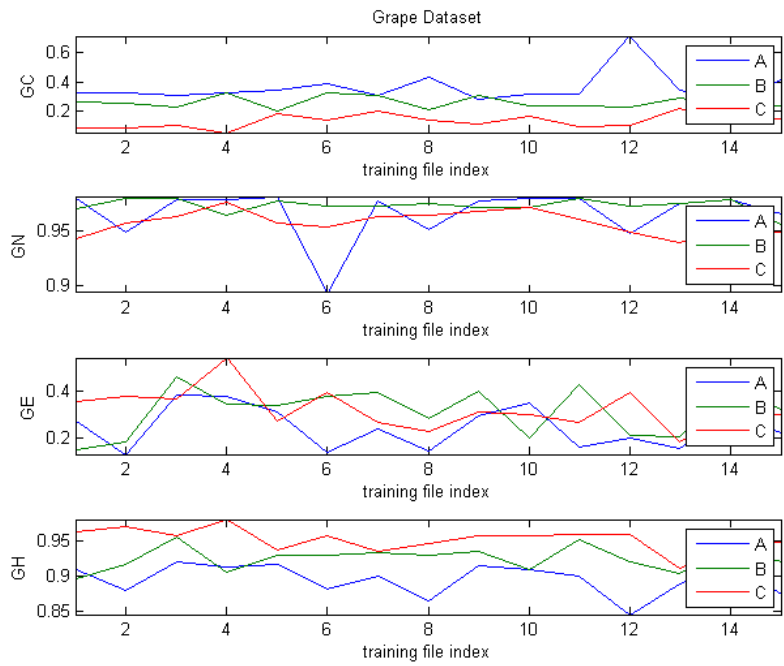

Figure 9: Variation of texture features for Grape training set 
The topology of the neural network used for classification of the sub-classes of the three fruits is shown in Fig. 10. It uses a 19-20-3 architecture i.e. 19 input nodes for the combined feature vector, 20 nodes in hidden layer and 3 output nodes for accommodating the three sub-classes A, B, C for each fruit, a tan-sigmoid activation function for the hidden layer, mean square error (MSE) threshold of 0.005 for convergence.

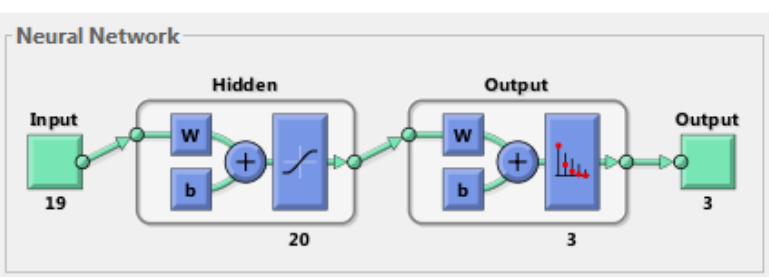

Figure 10: Topology of MLP classifier

Table 1 and Table 2 lists the classification accuracies using only Color and Texture features respectively.

Table 1. Percent Accuracies using Only Color Features

\begin{tabular}{|c|c|c|c|c|}
\hline $\begin{array}{c}\text { Fruit } \\
\text { Type }\end{array}$ & $\begin{array}{c}\text { Accuracy } \\
\text { \% } \\
\text { (Class A) }\end{array}$ & $\begin{array}{c}\text { Accuracy } \\
\text { \% } \\
\text { (Class B) }\end{array}$ & $\begin{array}{c}\text { Accuracy } \\
\text { \% } \\
\text { (Class C) }\end{array}$ & $\begin{array}{c}\text { Overall } \\
\text { Accuracy } \\
\text { \% }\end{array}$ \\
\hline Apple & 73.3 & 93.3 & 66.7 & 77.8 \\
\hline Mango & 53.3 & 73.3 & 93.3 & 73.3 \\
\hline Grape & 66.7 & 73.3 & 33.3 & 57.8 \\
\hline
\end{tabular}

Table 2. Percent Accuracies using Only Texture Features

\begin{tabular}{|c|c|c|c|c|}
\hline $\begin{array}{c}\text { Fruit } \\
\text { Type }\end{array}$ & $\begin{array}{c}\text { Accuracy } \\
\text { \% } \\
\text { (Class A) }\end{array}$ & $\begin{array}{c}\text { Accuracy } \\
\text { \% } \\
\text { (Class B) }\end{array}$ & $\begin{array}{c}\text { Accuracy } \\
\text { \% } \\
\text { (Class C) }\end{array}$ & $\begin{array}{c}\text { Overall } \\
\text { Accuracy } \\
\text { \% }\end{array}$ \\
\hline Apple & 93.3 & 93.3 & 100 & 95.6 \\
\hline Mango & 80 & 80 & 73.3 & 77.8 \\
\hline Grape & 80 & 86.7 & 93.3 & 86.7 \\
\hline
\end{tabular}

The accuracies obtained using a combination of color and texture as defined in Eq. (11), are shown in Table 3, which indicates the improvements achieved vis-à-vis sole features.

Table 3. Percent Accuracies using Combined Features

\begin{tabular}{|c|c|c|c|c|}
\hline $\begin{array}{c}\text { Fruit } \\
\text { Type }\end{array}$ & $\begin{array}{c}\text { Accuracy } \\
\text { \% } \\
\text { (Class A) }\end{array}$ & $\begin{array}{c}\text { Accuracy } \\
\text { \% } \\
\text { (Class B) }\end{array}$ & $\begin{array}{c}\text { Accuracy } \\
\text { \% } \\
\text { (Class C) }\end{array}$ & $\begin{array}{c}\text { Overall } \\
\text { Accuracy } \\
\text { \% }\end{array}$ \\
\hline Apple & 100 & 100 & 100 & 100 \\
\hline Mango & 93.3 & 93.3 & 86.7 & 91.1 \\
\hline Grape & 86.7 & 100 & 93.3 & 93.3 \\
\hline
\end{tabular}

Fig. 11, 12, 13 indicates the classification plots obtained from the outputs generated by the neural network.

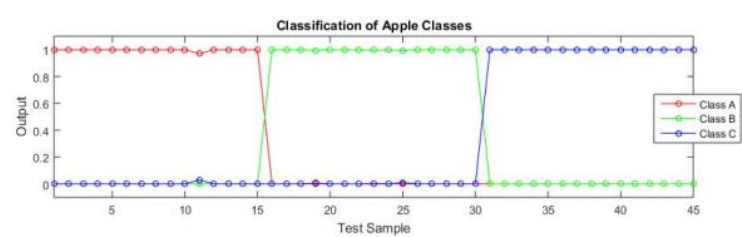

Figure 11: Classification Plots for Apple

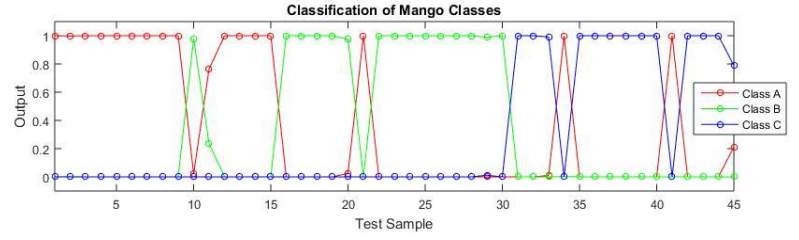

Figure 12: Classification Plots for Mango

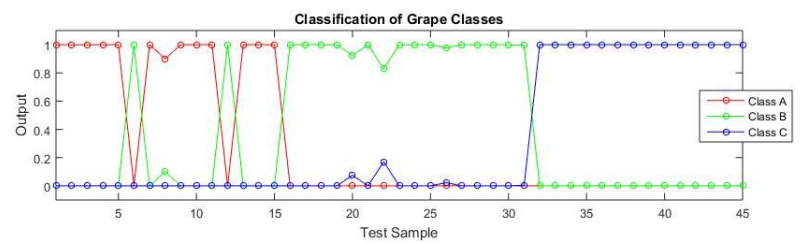

Figure 13: Classification Plots for Grape

\section{ANALYSIS}

From the previous section, it is evident that recognition performances have improved when features have been combined than when individual features have been used. It is to be noted that the classification is between sub-classes of fruits which display a lot of similarities between themselves as compared to recognition of different types of fruits. Fig. 14 summarizes the results indicating how the performance varies for the three types of fruits based on the features used.

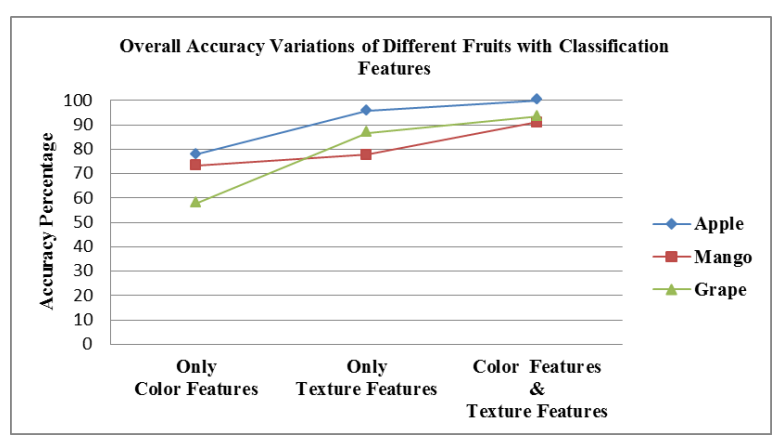

Figure 14: Overall Accuracy Variations of Different Fruit with Classification Feature

While most of the works reviewed here deals with inter-class recognition of fruits, in a previous work [1] intra-class recognition of fruits have been performed using contour shape features and Bayes Classifier. This approach has been implemented and executed on the current dataset. The results are shown in Table 4. A comparison between Table 3 and Table 4 indicates how the proposed approach compares with the approach in [1]. The reason for the lower performances of approach [1] is because of the shape characterization used there which tend to change with the viewing angle of the camera. Hence the present approach shows more robustness when viewing points are not constant.

Table 4. Percent accuracy using approach [1]

\begin{tabular}{|c|c|c|c|c|}
\hline $\begin{array}{c}\text { Fruit } \\
\text { Type }\end{array}$ & $\begin{array}{c}\text { Accuracy } \\
\text { \% } \\
\text { (Class A) }\end{array}$ & $\begin{array}{c}\text { Accuracy } \\
\text { \% } \\
\text { (Class B) }\end{array}$ & $\begin{array}{c}\text { Accuracy } \\
\text { \% } \\
\text { (Class C) }\end{array}$ & $\begin{array}{c}\text { Overall } \\
\text { Accuracy } \\
\text { \% }\end{array}$ \\
\hline Apple & 40 & 93.3 & 46.7 & 60 \\
\hline Mango & 46.7 & 86.7 & 66.7 & 66.7 \\
\hline Grape & 40 & 53.3 & 40 & 44.4 \\
\hline
\end{tabular}


Fig. 15 summarizes the performances of the two approaches. The blue line shows the present approach and the red line indicates approach [1]. Another important conclusion which can be drawn from the comparison is that shape based techniques are also not suitable when fruits are present in clusters or bunches like grapes. This is evident from the low $44 \%$ accuracy for approach in [1] compared to $93 \%$ for the present work where color and texture have been used instead.

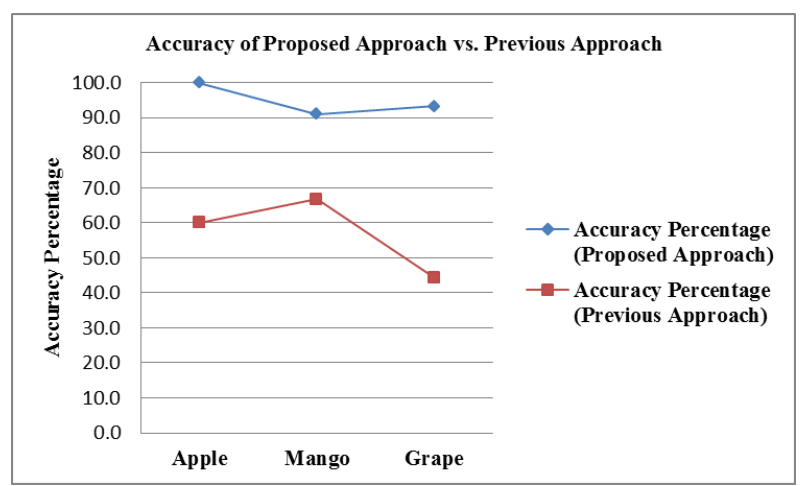

Figure 15: Accuracy of Proposed Approach vs. Previous Approach

\section{CONCLUSIONS \& FUTURE SCOPES}

This paper proposes a technique for intra-class classification of fruits using color and texture features. In general intra-class recognition is a more challenging problem than inter-class recognition since sub-types of a particular fruit are much more similar to each other than different types of fruits which can have largely dissimilar appearances. The color and texture features abstracted from three fruit types each with three subclasses have been demonstrated to produce acceptable results through experimentations on 270 color images. There are two main contributions of this work: (1) the proposed technique is not dependent on the camera viewpoint and provides acceptable results even when the angle between the camera and the object of interest changes (2) the proposed technique also shows robustness when fruits are present in a bunch or cluster and individual fruit shapes are difficult to extract.

Future work will involve proposing techniques to recognize unknown fruit classes and sub-classes as well as recognizing known fruit classes/sub-classes from within a collection of multiple fruits.

\section{REFERENCES}

[1] S. Mohamed Mansoor Roomi, R. Jyothi Priya, S.Bhumesh and P.Monisha, "Classification of Mangoes By Object Features and Contour Modeling", International Conference on Machine Vision and Image Processing (MVIP), IEEE, 14-15 Dec. 2012, pp. 165168.
[2] TheTimes of India, http://timesofindia.indiatimes.com/india/India-2ndlargest-fruit-producer-inworld/articleshow/50618234.cms

[3] Shiv Ram Dubey and Anand Singh Jalal, "Application of Image Processing in Fruit and Vegetable Analysis: A Review", Journal of Intelligent Systems, ISSN (Online) 2191-026X, vol. 24, issue 4, 2014, pp. 405-424.

[4] Hossam M. Zawbaa, Maryam Hazman, Mona Abbass and Aboul Ella Hassanien, "Automatic fruit classification using random forest algorithm", $14^{\text {th }}$ International Conference on Hybrid Intelligent Systems (HIS), IEEE, 14-16 Dec. 2014, pp. 164 - 168.

[5] Abdulhamid Haidar, Haiwei Dong and Nikolaos Mavridis, "Image-Based Date Fruit Classification", 4th International Congress on Ultra Modern Telecommunications and Control Systems and Workshops (ICUMT), IEEE, 3-5 Oct. 2012, pp. 357 363.

[6] Shiv Ram Dubey and A S Jalal, "Robust Approach for Fruit and Vegetable Classification", International Conference On Modeling Optimization and Computing, Elsevier, vol. 38, 2012, pp. 3449-3453.

[7] Md.Towhid Chowdhury, Md.Shariful Alam, Muhammad Asiful Hasan and Md.Imran Khan, "Vegetables detection from the glossary shop for the blind", IOSR Journal of Electrical and Electronics Engineering (IOSR-JEEE), eISSN: 2278-1676, p-ISSN: 2320-3331, vol. 8, issue 3 (Nov. - Dec. 2013), PP 43-53.

[8] Lv Jidong, Zhao De-An, Ji Wei and Ding Shihong, "Recognition of apple fruit in natural environment", Optik - International Journal for Light and Electron Optics, Elsevier, vol. 127, issue 3, February 2016, pp. 1354-1362.

[9] N. Otsu, "A threshold selection method from gray-level histograms", IEEE Trans on Sys. Man. Cyber. vol.9, issue 1, 1979, pp. 62-66.

[10] Rong Xiang, Yibin Ying and Huanyu Jiang, "Tests of a Recognition Algorithm for Clustered Tomatoes Based on Mathematical Morphology", 6th International Congress on Image and Signal Processing (CISP), IEEE, vol. 1, 16-18 Dec. 2013, pp. $464-468$

[11] Computers and Optics in Food Inspection, http://www.cofilab.com/portfolio/

[12] R. M. Haralick, "Statistical and structural approaches to texture”, Proc. IEEE, vol. 67, issue 5, 1979, pp. 786-804. 